\title{
SPECIAL VALUES OF $L$-SERIES
}

\author{
RHONDA L. HATCHER
}

(Communicated by William Adams)

\begin{abstract}
The main result of this paper is an equation representing the value of the Dirichlet series $L(f, A, s)$ at $s=k$ in terms of the height pairings of special points on a vector bundle $V$ associated with the quaternion algebra over $\mathbf{Q}$ ramified at $N$ and $\infty$.
\end{abstract}

\section{INTRODUCTION}

Let $N$ be a prime, $f \in S_{2 k}^{\text {new }}\left(\Gamma_{0}(N)\right)$, and $K$ an imaginary quadratic field of discriminant $-D$ where $N$ is inert. Let $A$ be an ideal class in $K$. In this paper, we will derive an equation representing the value of the Dirichlet series $L(f, A, s)$ at $s=k$ in terms of height pairings of special points on a vector bundle $V$ which is associated with the quaternion algebra over $\mathbf{Q}$ ramified at $N$ and $\infty$. The main identity is a generalization of the results found in [3] to the case where $D$ is composite, therefore the reader should refer to this paper for many of the details. The main identity has some interesting corollaries which can also be found in [3].

\section{The Dirichlet $L$-SERIES}

Set $\varepsilon=$ quadratic character of $(\mathbf{Z} / D \mathbf{Z})^{*}$ determined by $\varepsilon(p)=(-D / p)$ for $p \nmid D, 2 u=$ number of units in $K$, and set

$$
r_{A}(m)= \begin{cases}\text { number of ideals of norm } m \text { in } A, & \text { for } m \geq 1, \\ 1 /(2 u), & \text { for } m=0 .\end{cases}
$$

We will now define a Dirichlet series associated with $f$ and the ideal class $A$. Let $\sum_{m \geq 1} a_{m} q^{m}$, with $q=e^{2 \pi i z}$, be the Fourier expansion of $f$. Define the Dirichlet series $L(f, A, s)$ by

$$
L(f, A, s)=\sum_{\substack{m \geq 1 \\(m, N)=1}}\left(\frac{\varepsilon(m)}{m^{2 s-2 k+1}}\right) \sum_{m \geq 1}\left(\frac{a_{m} r_{A}(m)}{m^{s}}\right) .
$$

We will study the values of $L(f, A, s)$ at $s=k$ in the case $\varepsilon(N)=-1$.

Let $\mathscr{O}$ be the ring of integers in $K, h$ the class number of $K$, and $p$ a rational prime with $p \equiv-N(\bmod D)$. Then $p=\mathscr{I} \cdot \bar{I}$ is split in $\mathscr{O}$. Let

Received by the editors September 6, 1990.

1980 Mathematics Subject Classification (1985 Revision). Primary 11M99; Secondary 11G40. 
$\mathscr{A}$ be an ideal in the class of $A$. Then we say an ideal $\mathscr{B}$ is in the genus $\{-N A\}$ if $\mathscr{B} \mathscr{A} \mathscr{\mathcal { F }}$ has square class in $\operatorname{Pic}(\mathscr{O})$. Define $R_{\{-N A\}}(m)$ to be the number of integral ideals in $\{-N A\}$ of norm $m$. Note that since two ideals with the same norm lie in the same genus, $R_{\{-N A\}}(m)$ is equal to $R(m)$ or zero where

$$
R(m)= \begin{cases}\text { number of ideals of } \mathscr{O} \text { of norm } m, & \text { for } m \geq 1, \\ h /(2 u), & \text { for } m=0 .\end{cases}
$$

In particular, in the case $D$ prime $R_{\{-N A\}}(m)=R(m)$ for all $m$. Define $\delta(m)$ to be $2^{k}$, where $k$ is the number of prime factors of $(m, D)$. Let $P_{n}(x)$ be the Legendre polynomial of degree $n$. We are now ready to state the following result which is proved in [2, p. 291, Theorem 5.6].

Theorem 2.2. Let $\varepsilon(N)=-1$ and let $k$ be any integer $\geq 1$. For $m \geq 0$, define

$$
\begin{aligned}
b_{m, A}=(m D)^{k-1} u^{2}\left[r_{A}(m D) \frac{h}{u}+\right. & \sum_{0<n \leq m D / N} \delta(n) r_{A}(m D-n N) \\
& \left.\quad \times R_{\{-N A\}}(n) P_{k-1}\left(1-\frac{2 n N}{m D}\right)\right] .
\end{aligned}
$$

Then $\sum_{m>0} b_{m, A} q^{m}$ is a modular form of weight $2 k$ and level $N$, and a cusp form if $k \neq 1$, and

$$
L(f, A, k)=\frac{2^{2 k-2}(k-1) !}{(2 k-2) !} \frac{1}{D^{k-1 / 2} u^{2}}\left(f, \sum_{m \geq 0} b_{m, A} q^{m}\right),
$$

where $\left(f, \sum_{m \geq 0} b_{m, A} q^{m}\right)$ is the Petersson product of $f$ and $\sum_{m \geq 0} b_{m, A} q^{m}$.

We will make use of the result of Theorem 2.2 to derive an alternative representation for the values $L(f, A, k)$.

\section{THE VECTOR BUNDLE $V$ AND THE HEIGHT PAIRING}

Let $B$ be the quaternion algebra over $\mathbf{Q}$ ramified at the rational prime $N$ and at $\infty$, and let $R$ be a maximal order of $B$. Let $\widehat{\mathbf{Z}}=\lim \mathbf{Z} / n \mathbf{Z}=\prod_{p} \mathbf{Z}_{p}$ be the profinite completion of $\mathbf{Z}$, and let $\widehat{\mathbf{Q}}=\widehat{\mathbf{Z}} \otimes \mathbf{Q}=\coprod_{p} \mathbf{Q}_{p}$ be the ring of finite adèles of $\mathbf{Q}$. Set $B_{p}=B \otimes \mathbf{Q}_{p}$ and $R_{p}=R \otimes \mathbf{Z}_{p}$. Let $\widehat{B}=B \otimes \widehat{\mathbf{Q}}=\coprod_{p} B_{p}$ and $\widehat{R}=R \otimes \widehat{\mathbf{Z}}=\prod_{p} R_{p}$.

The quaternion algebra $B$ corresponds to an algebraic curve $Y$ of genus zero over $\mathbf{Q}$ as described in [3, p. 541]. Let $W_{2 k-1}$ be the inner product space $\operatorname{Sym}^{2 k-2}(W)$, where $W=\mathbf{C} x \oplus \mathbf{C} y$ is the two dimensional representation of $S U(2)$ with $[x, x]=[y, y]=1$ and $[x, y]=0$. Then the vector bundle $V$ is defined by $V=\widehat{R}^{*} \backslash \widehat{B}^{*} \times Y \times W_{2 k-1} / B^{*}$.

We now define $\operatorname{Pic}(V)$. Let $\left\{I_{1}, I_{2}, \ldots, I_{n}\right\}$ be a set of left ideals of $R$ which represent the distinct ideal classes. The set $\left\{I_{1}, I_{2}, \ldots, I_{n}\right\}$ corresponds to a choice of elements $g_{1}, g_{2}, \ldots, g_{n}$ in $\widehat{R}^{*} \backslash \widehat{B}^{*}$ such that $\widehat{B}^{*}=$ $\bigcup_{i=1}^{n} \widehat{R}^{*} g_{i} B^{*}$. Let $R_{i}$ denote the right order of $I_{i}$. For each $\Gamma_{i}=R_{i} /\langle \pm 1\rangle$, define $W^{\Gamma_{i}}=\left\{w \in W: \gamma(w)=w\right.$ for all $\left.\gamma \in \Gamma_{i}\right\}$. Then $\operatorname{Pic}(V)=\bigoplus_{i=1}^{n} W^{\Gamma_{i}}$. 
Any $v \in V$ is equivalent to the double coset $\left(g_{i}, y, w\right)$ for some $i$. Define the class of the point $v$ to be the projection of $w$ in $W^{\Gamma_{i}}$ given by $\operatorname{class}(v)=\left(\left|\Gamma_{i}\right|\right)^{-1} \sum_{\gamma \in \Gamma_{i}} \gamma(w)$.

A height pairing $\langle$,$\rangle mapping V \times V$ into $\mathbf{Q}$ can be defined as follows. If $v_{1}=g \times \widetilde{y_{1}} \times \widetilde{w_{1}} \cong g_{i} \times y_{1} \times w_{1}$ and $v_{2}=h \times \widetilde{y_{2}} \times \widetilde{w_{2}} \cong g_{j} \times y_{2} \times w_{2}$, then

$$
\left\langle v_{1}, v_{2}\right\rangle= \begin{cases}0, & \text { if } i \neq j, \\ \sum_{\gamma \in \Gamma_{i}}\left[w_{1}, \gamma\left(w_{2}\right)\right]_{W_{2 k-1}}, & \text { if } i=j .\end{cases}
$$

For the remainder of the paper, we fix $K$ an imaginary quadratic field of discriminant $-D$ where the prime $N$ is inert, and $\mathscr{O}$ the ring of integers of $K$.

Let $X=\widehat{R}^{*} \backslash \widehat{B}^{*} \times Y / B^{*}$. There exists a canonical identification $Y(K) \simeq$ $\operatorname{Hom}(K, B)$ as described in [3, p. 541]. The special points of $V$ of discriminant $-D$ are defined to be points of the form $v=g \times y \times w_{0}$, where $w_{0}$ is a special element of $W_{2 k-1}$ as defined in [3, p. 545], $g \times y$ lies in the image of $\widehat{R}^{*} \backslash \widehat{B}^{*} \times Y(K)$ in $X(K)$, and the embedding $f$ corresponding to $y$ satisfies $f(K) \cap g^{-1} \widehat{R} g=f\left(\mathscr{O}_{-D}\right)$, where $\mathscr{O}_{-D}$ is the order of discriminant $-D$. An action of $\operatorname{Pic}(\mathscr{O})$ on the special points of $V$ of discriminant $-D$ given by $v \rightarrow v_{a}$ for $a \in \operatorname{Pic}(\mathscr{O})$ is defined in [3, p. 545].

Our main identity is an equation representing the value of $L(f, A, k)$ in terms of the values of the height pairings of special points on $V$.

\section{The MAIN IDENTITY}

A correspondence $t_{m}$ can be defined on $V=\widehat{R}^{*} \backslash \widehat{B}^{*} \times Y \times W_{2 k-1} / B^{*}$ as follows. For $p \neq N, R_{p}^{*} \backslash B_{p}^{*} \cong G L_{2}\left(\mathbf{Z}_{p}\right) \backslash G L_{2}\left(\mathbf{Q}_{p}\right)$ which index lattices in $\mathbf{Q}_{p}^{2}$, and locally, $t_{m}$ maps a lattice $L$ in $G L_{2}\left(\mathbf{Z}_{p}\right) \backslash G L_{2}\left(\mathbf{Q}_{p}\right)$ to the sum of all sublattices of index $p^{\operatorname{ord}_{p}(m)}$. For $p=N, R_{p}^{*} \backslash B_{p}^{*} \cong \mathbf{Z}$, and locally, $t_{m}$ maps a coset $A$ into $\pi^{\operatorname{ord}_{p}(m)} A$, where $\pi$ is a uniformizing parameter in $R_{p}$. This action of $t_{m}$ on $\widehat{R}^{*} \backslash \widehat{B}^{*}$ induces a correspondence on $V$

$$
t_{m}(g \times y \times w)=\sum_{h \in t_{m}(g)}(h \times y \times w) .
$$

The correspondences $t_{m}$ on $V$ induce endomorphisms of $\operatorname{Pic}(V)=\bigoplus_{i=1}^{n} W^{\Gamma_{i}}$. With a particular choice of basis, the action of $t_{m}$ on $\operatorname{Pic}(V)$ is given by $B_{2 k-2}^{t}(m)$, the transpose of the Brandt matrix $B_{2 k-2}(m)$ [3, pp. 545-546].

Define the series $\phi(v, w), v, w \in \operatorname{Pic}(V)$, as follows

$$
\begin{aligned}
& \text { For } k>1, \quad \phi(v, w)=\sum_{m \geq 1}\left\langle v, t_{m} w\right\rangle q^{m}, \\
& \text { For } k=1, \quad \phi(v, w)=\frac{\operatorname{deg} v \cdot \operatorname{deg} w}{2}+\sum_{m \geq 1}\left\langle v, t_{m} w\right\rangle q^{m} .
\end{aligned}
$$

It can be shown that for any $v, w \in \operatorname{Pic}(V), \phi(v, w)$ is a modular form of weight $2 k$ for $\Gamma_{0}(N)$ and if $s>0$, it is a cusp form [3, p. 548, Proposition 5.3].

Let $v$ be a fixed point of discriminant $-D$ on $V$, and $A$ be an ideal class in $K$. Define $g_{A}=\sum_{B} \phi\left(v_{B}, v_{A B}\right)$, where the sum is over all classes $B$ in $\operatorname{Pic}(\mathscr{O})$. Then $g_{A}$ is a modular form of weight $2 k$ on $\Gamma_{0}(N)$. 
The main identity is

Proposition 4.1. For $\varepsilon(N)=-1$ and $k$ any integer $\geq 1$,

$$
L(f, A, k)=\frac{\left(f, g_{A}\right)}{u^{2} D^{k-1 / 2}(k-1) !^{2}} .
$$

This identity relates the central critical values of $L(f, A, s)$ with height pairings on $\operatorname{Pic}(V)$. To prove Proposition 4.1, we will first need to compute $\left\langle v_{B}, t_{m} v_{A B}\right\rangle$ for $m \geq 1$, where $v$ is a fixed point on $V$ of discriminant $-D$ and $A$ and $B$ lie in $\operatorname{Pic}(\mathscr{O})$.

Write $v=x \times w_{0}, v_{B}=x_{B} \times w_{0}$, and $v_{A B}=x_{A B} \times w_{0}$, so that $x_{B}$ and $x_{A B}$ are special points of discriminant $-D$ on $X$. Each component $X_{i}=Y / \Gamma_{i}$ of the curve $X$ is indexed by a supersingular elliptic curve of characteristic $N$. Suppose $E$ is the elliptic curve corresponding to $x_{A B}$ and $E^{\prime}$ the elliptic curve corresponding to $x_{B}$. We will denote the group $\operatorname{Hom}\left(E, E^{\prime}\right)$ by $\operatorname{Hom}\left(x_{A B}, x_{B}\right)$.

Let $\mathscr{D}=(\sqrt{-D})$ be the different ideal of $\mathscr{O}$. Let $\mathscr{A}$ and $\mathscr{B}$ be ideals in the classes of $A$ and $B$ respectively, which are relatively prime to $\mathscr{D}$. Fix one solution $\varepsilon$ to the equation $\varepsilon^{2} \equiv-N \bmod D$. Then, in the case $D$ prime, we have the following result which is proved in [3, p. 550, Proposition 7.1].

Lemma 4.2. Suppose $D$ is prime. Then there exists a bijection

$$
\operatorname{Hom}\left(x_{A B}, x_{B}\right) \leftrightarrow\left\{\alpha+\beta j: \alpha \in \mathscr{D}^{-1} \mathscr{A}, \beta \in \mathscr{D}^{-1} \frac{\overline{\mathscr{B}}}{\mathscr{\mathscr { B }}} \overline{\mathscr{A}}, \alpha \equiv \varepsilon \beta \bmod \mathscr{O}\right\} .
$$

If $\varphi \in \operatorname{Hom}\left(x_{A B}, x_{B}\right)$ corresponds to $\alpha+\beta j$, then $\operatorname{deg} \varphi=(\mathbf{N} \alpha+N \mathbf{N} \beta) / \mathbf{N} \mathscr{A}$.

In the case when $D$ is composite, let $p$ be a rational prime with $p \equiv$ $-N \bmod D$. Then $p=\mathscr{I} \cdot \overline{\mathscr{I}}$ is split in $K$ and $B=K+K j$ with $j^{2}=-N p$ and $j \alpha=\bar{\alpha} j$ for all $\alpha \in K$. In this case, we have the following bijection.

$$
\begin{aligned}
& \operatorname{Hom}\left(x_{A B}, x_{B}\right) \leftrightarrow\left\{\alpha+\beta j: \alpha \in \mathscr{D}^{-1} \mathscr{A},\right. \\
& \left.\qquad \beta \in \mathscr{D}^{-1} \mathscr{I}^{-1} \frac{\overline{\mathscr{B}}}{\mathscr{\mathscr { B }}} \overline{\mathscr{A}}, \alpha \equiv \varepsilon \beta \bmod \mathscr{O}\right\} .
\end{aligned}
$$

It is also shown in [3, pp. 551-554] that for $D$ prime

$$
\left\langle v_{B}, t_{m} v_{A B}\right\rangle=\frac{1}{2} \sum_{\substack{\phi \in \operatorname{Hom}\left(x_{A B}, x_{B}\right)_{\operatorname{deg} m} \\ \phi \leftrightarrow b=\alpha+\beta j, \mathbf{N} b=m \mathbf{N} \mathscr{A}}} m^{k-1} P_{k-1}\left(\frac{\alpha \bar{\alpha}-N \beta \bar{\beta}}{\alpha \bar{\alpha}+N \beta \bar{\beta}}\right)\left[w_{0}, w_{0}\right] .
$$

In the case when $D$ is composite and $p$ is as defined above, it can be shown by using a computation analogous to that used to derive (4.4) that

$$
\left\langle v_{B}, t_{m} v_{A B}\right\rangle=\frac{1}{2} \sum_{\substack{\phi \in \operatorname{Hom}\left(x_{A B}, x_{B}\right)_{\operatorname{deg} m} m \\ \phi \leftrightarrow b=\alpha+\beta j, \mathbf{N} b=m \mathbf{N} \mathscr{A}}} m^{k-1} P_{k-1}\left(\frac{\alpha \bar{\alpha}-p N \beta \bar{\beta}}{\alpha \bar{\alpha}+p N \beta \bar{\beta}}\right)\left[w_{0}, w_{0}\right] .
$$


Applying the correspondence in equation (4.3) to the result of (4.5), we get (4.6)

$$
\begin{aligned}
& \left\langle v_{B}, t_{m} v_{A B}\right\rangle=\frac{1}{2} \sum_{\substack{\alpha+\beta j \\
\mathbf{N}(\alpha+\beta j)=m \mathbf{N} \mathscr{A}}} m^{k-1} P_{k-1}\left(\frac{\alpha \bar{\alpha}-p N \beta \bar{\beta}}{\alpha \bar{\alpha}+p N \beta \bar{\beta}}\right)\left[w_{0}, w_{0}\right] . \\
& \begin{array}{c}
\alpha \in \mathscr{D}^{-1} \mathscr{A}, \beta \in \mathscr{D}^{-1} \mathscr{I}^{-1} \frac{\overline{\mathscr{T}}}{\mathscr{\mathscr { Q }}} \overline{\mathscr{A}} \\
\alpha \equiv \varepsilon \beta \bmod \mathscr{O}
\end{array}
\end{aligned}
$$

To complete the computation of $\left\langle v_{B}, t_{m} v_{A B}\right\rangle$ in the case $D$ composite with $p$ as defined above, we consider the ideals $\mathscr{C}=(\alpha) \mathscr{D}^{-1}$ and $\mathscr{C}^{\prime}=$ $(\beta) \mathscr{D} \mathscr{S} \mathscr{B} \mathscr{\mathscr { B }}-1 \overline{\mathscr{A}}^{-1}$, which satisfy the identity

$$
\mathbf{N} \mathscr{C}+N \mathbf{N} \mathscr{C}^{\prime}=m D \text {. }
$$

Let $n=\mathbf{N} \mathscr{C}^{\prime}$. Then $\mathbf{N} \mathscr{C}=m D-n N$. We have $\mathbf{N} \mathscr{C}^{\prime}=(\mathbf{N} \beta) D p(1 / \mathbf{N} \mathscr{A})$, and hence $\mathbf{N} \beta=(n / D p) \mathbf{N} \mathscr{A}$. Since $\mathbf{N} \mathscr{C}=m D-n N=(\mathbf{N} \alpha) D(1 / \mathbf{N} \mathscr{A})$, it follows that $\mathbf{N} \alpha=((m D-n N) / D) \mathbf{N} \mathscr{A}$. Therefore, when $n=\mathbf{N} \mathscr{C}^{\prime}$,

$$
\begin{aligned}
\frac{\alpha \bar{\alpha}-p N \beta \bar{\beta}}{\alpha \bar{\alpha}+p N \beta \bar{\beta}} & =\frac{\mathbf{N} \alpha-p N \mathbf{N} \beta}{\mathbf{N} \alpha+p N \mathbf{N} \beta}=\frac{((m D-n N) / D) \mathbf{N} \mathscr{A}-p N(n / D p) \mathbf{N} \mathscr{A}}{((m D-n N) / D) \mathbf{N} \mathscr{A}+p N(n / D p) \mathbf{N} \mathscr{A}} \\
& =\frac{m D-2 n N}{m D}=1-\frac{2 n N}{m D}
\end{aligned}
$$

The ideal $\mathscr{C}$ lies in the class of $A^{-1}$, and the ideal $\mathscr{C}^{\prime}$ lies in the class of $A \mathscr{I} B^{2}$. Therefore, the number of solutions to (4.7) with $\mathbf{N} \mathscr{C}^{\prime}=n$ is given by

$$
\begin{array}{ll}
r_{A^{-1}}(m D-n N) r_{A \mathscr{F} B^{2}}(n), & \text { if } n \neq 0, \frac{m D}{N}, \\
r_{A^{-1}}(m D), & \text { if } n=0, \\
r_{A \mathscr{I}^{2}}\left(\frac{m D}{N}\right), & \text { if } n=\frac{m D}{N} .
\end{array}
$$

Each solution $\mathscr{C}, \mathscr{C}^{\prime}$ to (4.7) corresponds to a solution to the identity

$$
\begin{gathered}
\mathbf{N}(\alpha+\beta j)=m \mathbf{N} \mathscr{A}, \\
\alpha \in \mathscr{D}^{-1} \mathscr{A}, \quad \beta \in \mathscr{D}^{-1} \mathscr{J}^{-1} \frac{\overline{\mathscr{B}}}{\mathscr{\mathscr { B }}} \overline{\mathscr{A}}, \\
\alpha \equiv \varepsilon \beta \bmod \mathscr{O} .
\end{gathered}
$$

Each pair $\mathscr{C}, \mathscr{C}^{\prime}$ with $\mathbf{N} \mathscr{C}^{\prime}=n \neq 0,(n D) / N$, so that $\mathbf{N} \mathscr{C} \neq 0$, gives $(2 u)^{2}$ candidates $(\alpha, \beta)$ for solutions to (4.9). If $n \equiv 0 \bmod D$, then all of these candidates turn out to be solutions since $\alpha \equiv \varepsilon \beta \bmod \mathscr{O}$ is satisfied. If $n \not \equiv 0 \bmod D$, then either $\alpha \equiv \varepsilon \beta \bmod \mathscr{O}$ or $\alpha \equiv-\varepsilon \beta \bmod \mathscr{O}$, but not both. Hence, only one half of the candidates are actually solutions to (4.9). Summarizing this information, we see that the number of solutions to the identity (4.9) is given by

$$
\begin{array}{ll}
(2 u)^{2}\left(\frac{1}{2}\right) \delta(n) r_{A^{-1}}(m D-n N) r_{A \mathscr{S}^{2}}(n), & \text { if } n \neq 0, \frac{m D}{N} \\
(2 u) r_{A^{-1}}(m D), & \text { if } n=0, \\
(2 u) r_{A \mathscr{I} B^{2}}\left(\frac{m D}{N}\right), & \text { if } n=\frac{m D}{N} .
\end{array}
$$


Using the convention $r_{A}(0)=1 / 2 u$ for any ideal class $A$, the number of solutions to the identity (4.9) is given by

$$
2 u^{2} \delta(n) r_{A^{-1}}(m D-n N) r_{A \mathscr{J} B^{2}}(n) .
$$

Hence, using the result of $(4.10)$, the fact that $(\alpha \bar{\alpha}-p N \beta \bar{\beta}) /(\alpha \bar{\alpha}+p N \beta \bar{\beta})=$ $1-(2 n N) /(m D)$ when $\mathbf{N} \mathscr{C}^{\prime}=n$, and (4.6) we have

$$
\begin{aligned}
\left\langle v_{B}, t_{m} v_{A B}\right\rangle= & \frac{1}{2} \sum_{0 \leq n \leq m D / N} 2 u^{2} \delta(n) r_{A^{-1}}(m D-n N) r_{A \mathscr{J}_{B^{2}}}(n) m^{k-1} \\
& \times P_{k-1}\left(1-\frac{2 n N}{m D}\right)\left[w_{0}, w_{0}\right] \\
= & u^{2} m^{k-1}\left[w_{0}, w_{0}\right] \sum_{0 \leq n \leq m D / N} \delta(n) r_{A^{-1}}(m D-n N) \\
& \times r_{A \mathscr{J} B^{2}}(n) P_{k-1}\left(1-\frac{2 n N}{m D}\right) .
\end{aligned}
$$

In $[3$, p. 556] it is shown that

$$
\left[w_{0}, w_{0}\right]=\frac{2^{2 k-2} D^{k-1}(k-1) !^{3}}{(2 k-2) !} .
$$

We have now completed the computation of $\left\langle v_{B}, t_{m} v_{A B}\right\rangle$, and we are ready to prove the following.

Lemma 4.13. For all $m \geq 1$,

$$
\begin{aligned}
& \sum_{B}\left\langle v_{B}, t_{m} v_{A B}\right\rangle=\frac{u^{2}(m D)^{k-1} 2^{2 k-2}(k-1) !^{3}}{(2 k-2) !} \\
& \times\left(r_{A}(m D)\left(\frac{h}{u}\right)+\sum_{0<n \leq m D / N} \delta(n) r_{A}(m D-n N) R_{\{-N A\}}(n) P_{k-1}\left(1-\frac{2 n N}{m D}\right)\right),
\end{aligned}
$$

where the sum is over all classes $B$ in $\operatorname{Pic}(\mathscr{O})$.

Proof. Applying (4.11) and (4.12) and the fact that $r_{A^{-1}}(k)=r_{A}(k)$, we have

$$
\begin{aligned}
\sum_{B}\left\langle v_{B}, t_{m} v_{A B}\right\rangle \\
=\sum_{B} u^{2} m^{k-1}\left(\frac{2^{2 k-2} D^{k-1}(k-1) !^{3}}{(2 k-2) !}\right) \\
\quad \times \sum_{0 \leq n \leq m D / N} \delta(n) r_{A}(m D-n N) r_{A \mathscr{J}} B^{2}(n) P_{k-1}\left(1-\frac{2 n N}{m D}\right) \\
=\frac{u^{2}(m D)^{k-1} 2^{2 k-2}(k-1) !^{3}}{(2 k-2) !} \\
\quad \times \sum_{B} \sum_{0 \leq n \leq m D / N} \delta(n) r_{A}(m D-n N) r_{A \mathscr{J} B}(n) P_{k-1}\left(1-\frac{2 n N}{n D}\right) .
\end{aligned}
$$

The sum over all classes $B$ only gives the ideals in the genus $\{A \mathscr{I}\}=\{-N A\}$. and hence the result follows.

If $b_{m, A}$ is as defined in Theorem 2.2 and we apply the result of Lemma 4.13, we immediately arrive at the following. 


\section{Corollary 4.14.}

$$
b_{m, A}=\frac{(2 k-2) !}{2^{2 k-2}(k-1) !^{3}} \sum_{B}\left\langle v_{B}, t_{m} v_{A B}\right\rangle
$$

We are now ready to prove the main identity, Proposition 4.1. By Theorem 2.2 and Corollary 4.14, we have for $\varepsilon(N)=-1$ and $k$ any integer $\geq 1$,

$$
\begin{aligned}
L(f, & A, k)=\frac{2^{2 k-2}(k-1) !}{(2 k-2) !} \frac{1}{D^{k-1 / 2} u^{2}}\left(f, \sum_{m \geq 0} b_{m, A} q^{m}\right) \\
& =\frac{2^{2 k-2}(k-1) !}{(2 k-2) !} \frac{1}{D^{k-1 / 2} u^{2}}\left(f, \sum_{m \geq 0}\left(\frac{(2 k-2) !}{2^{2 k-2}(k-1) !^{3}} \sum_{B}\left\langle v_{B}, t_{m} v_{A B}\right\rangle\right) q^{m}\right) \\
& =\frac{1}{u^{2} D^{k-1 / 2}(k-1) !^{2}}\left(f, \sum_{B}\left(\sum_{m \geq 0}\left\langle v_{B}, t_{m} v_{A B}\right\rangle q^{m}\right)\right) .
\end{aligned}
$$

For $k=1$, the constant term in $g_{A}$ is given by $h / 2=u^{2} b_{0, A}$. Hence,

$$
L(f, A, k)=\frac{\left(f, g_{A}\right)}{u^{2} D^{k-1 / 2}(k-1) !^{2}} .
$$

This completes the proof of Proposition 4.1.

\section{REFERENCES}

1. B. H. Gross, Heights and the special values of $L$-series, CMS Conf. Proc. 7 (1986).

2. B. H. Gross and D. Zagier, Heegner points and derivatives of L-series, Invent. Math. 84 (1986), 225-320.

3. R. L. Hatcher, Heights and L-series, Canad. J. Math. 42 (1990), 533-560.

Department of Mathematics, Texas Christian University, Fort Worth, Texas 76129 